\title{
An in vitro investigation of species-dependent intestinal transport of selenium and the impact of this process on selenium bioavailability
}

\author{
Celine Thiry ${ }^{1}$, Ann Ruttens ${ }^{2}$, Luc Pussemier ${ }^{2}$ and Yves-Jacques Schneider ${ }^{1 *}$ \\ ${ }^{1}$ Biochimie Cellulaire, Nutritionnelle \& Toxicologique, Institut des Sciences de la Vie E UCLouvain, Croix du Sud, \\ 5, B1348 Louvain-la-Neuve, Belgium \\ ${ }^{2}$ CODA-CERVA-VAR Veterinary and Agrochemical Research Centre, Leuvensesteenweg, 17, B3080 Tervuren, Belgium
}

(Submitted 8 June 2012 - Final revision received 20 August 2012 - Accepted 24 August 2012 - First published online 13 November 2012)

\begin{abstract}
A range of Se species has been shown to occur in a variety of different foodstuffs. Depending on its speciation, Se is more or less bioavailable to human subjects. In the present study, the role of speciation as a determinant of Se bioavailability was addressed with an investigation of species-specific mechanisms of transport at the intestinal level. The present work focused on four distinct Se compounds (selenate (Se(VI)), selenite (Se(IV)), selenomethionine (SeMet) and methylselenocysteine (MeSeCys)), whose intestinal transport was mimicked through an in vitro bicameral model of enterocyte-like differentiated Caco-2 cells. Efficiency of Se absorption was shown to be species dependent (SeMet $>$ MeSeCys $>$ Se(VI) $>$ Se(IV)). In the case of SeMet, MeSeCys and Se(VI), the highly polarised passage from the apical to basolateral pole indicated that a substantial fraction of transport was transcellular, whilst results for Se(IV) indicated paracellular diffusion. Passage of the organic Se species (SeMet and MeSeCys) became saturated after $3 \mathrm{~h}$, but no such effect was observed for the inorganic species. In addition, SeMet and MeSeCys transport was significantly inhibited by their respective $\mathrm{S}$ analogues methionine and methylcysteine, which suggests a common transport system for both kinds of compounds.
\end{abstract}

Key words: Selenium: Selenium species: Intestinal absorption: Transport mechanisms

Se is a micronutrient whose essentiality for human subjects and animals has been recognised for over 50 years $^{(1)}$. Essentiality of this element is associated with its presence in the active centre of several antioxidant enzymes ${ }^{(2)}$, allowing Se to play a critical role in the cellular redox balance. Se is also involved in thyroid regulation ${ }^{(3)}$, keratinocyte function, skin development $^{(4)}$ and heavy metal detoxification ${ }^{(5)}$. Furthermore, specific anti-cancer properties have been attributed to methylselenol, an intermediate in Se metabolism ${ }^{(6-8)}$. Low Se intake causes deficiency symptoms, ranging from brittle hair and nails $^{(4)}$ to serious cardiac and osteoarthritic pathologies ${ }^{(5,9)}$. On the other hand, excessive Se intake may cause possibly fatal intoxications in severe cases ${ }^{(9,10)}$. The optimal range of Se intake is narrow, and corresponds to $60-70 \mu \mathrm{g} / \mathrm{d}^{(11)}$.

Se enters the body almost exclusively through food. Depending on the type of food, both concentration and chemical form of Se can vary. These two factors (dose and speciation) critically influence Se bioavailability, bioactivity and potential toxicity ${ }^{(12)}$. Several studies dealing with Se dietary enrichment of human subjects and animals have indicated that organic forms are more bioavailable and less toxic than inorganic ones ${ }^{(13-18)}$. In rats, Se concentrations in blood and liver were reported to be up to four times higher following SeMet supplementation compared with selenite (Se(IV)) supplementation $^{(14)}$. Similarly, in human subjects, plasma Se content was twice as high after SeMet enrichment compared with Se(IV) addition ${ }^{(17,18)}$. In crucian carps, the selenoenzyme glutathione peroxidase was expressed more after SeMet intake than after comparable Se(IV) intake ${ }^{(15)}$.

In the present study, an in vitro model of the intestinal barrier was used for a detailed investigation of bioavailability of four Se species (selenate (Se(VI)), Se(IV), selenomethionine (SeMet) and methylselenocysteine (MeSeCys)), as well as for the study of the mechanisms involved in their transport across the intestinal epithelium. Bioavailability - or the bioavailable fraction - is considered to be the fraction of an element that is absorbed through intestinal membrane and reaches the systemic circulation, from where it can be distributed to organs and tissues and possibly become bioactive ${ }^{,(19)}$. We worked with enterocyte-like-differentiated Caco- 2 cells in

Abbreviations: AP, apical; BL, basolateral; DMEM, Dulbecco's modified Eagle's medium; EGTA, ethylene glycol tetraacetic acid; FBS, fetal bovine serum; HBSS, Hank's buffered salt solution; LY, lucifer yellow; MeSeCys, methylselenocysteine; $P_{\text {app }}$, apparent permeability; Se(IV), selenite; Se(VI), selenate; SeMet, selenomethionine; TEER, transepithelial electrical resistance.

*Corresponding author: Y.-J. Schneider, fax +32 10 474895, email yves-jacques.schneider@uclouvain.be 
a culture system that mimicked Se transport through the intestinal membrane, and we measured the degree of absorption of the aforementioned Se species. In addition, the occurrence of para- and transcellular pathways was investigated, as well as the possible competition for absorption between Se species and their S analogues. Given the high sensitivity of the detection method (inductively coupled plasma-MS), we were able to work with realistic concentrations of Se intake and to perform speciation analyses on the products of transport experiments. In this way, the present study aimed at refining knowledge of the influence of the mode of transport of Se on its species-dependent bioavailability.

\section{Materials and methods \\ Cell culture system}

Caco-2 cells, originating from a human colorectal adenocarcinoma, were obtained from the American Type Culture Collection (ATCC HTB-37). Cells were stored in liquid $\mathrm{N}_{2}$ and aliquots were regularly defrosted for experiments. Caco-2 cells were cultured at $37^{\circ} \mathrm{C}$ in a humidified atmosphere of $5 \% \mathrm{CO}_{2}-95 \%$ air (v/v) in Dulbecco's modified Eagle's medium (DMEM; Lonza) supplemented with $10 \%(\mathrm{v} / \mathrm{v})$ heatinactivated fetal bovine serum (FBS; HyClone; PerbioSciences), $1 \%(\mathrm{v} / \mathrm{v})$ non-essential amino acids (Lonza) and $1 \%(\mathrm{v} / \mathrm{v})$ L-glutamine $200 \mathrm{~mm}$ (Lonza). Cells were expanded in $175 \mathrm{~cm}^{2}$ flasks and sub-cultured at $90 \%$ confluence with a trypsin-EDTA solution (Lonza). Each sub-cultivation incremented by one the passage number and cells were used for experiments until passage number 60. After sub-cultivation, cells were seeded on permeable PE Transwell ${ }^{\circledR}$ cell culture inserts (24 mm diameter, $0.4 \mu \mathrm{m}$ pore size; Corning; Elscolab) at a density of $4 \times 10^{5}$ cells per insert. Inserts were placed in sixwell plates (Corning; Elscolab), delineating upper (apical, $\mathrm{AP}$ ) and lower (basolateral, BL) compartments. AP and $\mathrm{BL}$ compartments were filled, respectively, with 1.5 and $2.5 \mathrm{ml}$ of culture medium that was changed three times a week until full differentiation of cells ( $21 \mathrm{~d}$ post-seeding). Because serum contains traces of Se, the last medium change before starting the experiment used $0.5 \%$ FBS instead of $10 \%$.

\section{Monitoring of the cell culture system}

Several cell culture parameters were monitored to guarantee the suitability and reproducibility of the model. Integrity of the cell monolayer and the effectiveness of the epithelial tight junctions were verified prior to and after experiments by measuring the transepithelial electrical resistance (TEER; Endohm 24, World Precision Instruments). The absence of Se influence on the functioning of tight junctions was assessed by measuring the permeability to $500 \mu \mathrm{g} / \mathrm{ml}$ lucifer yellow (LY; Sigma-Aldrich) in the presence or absence of Se species, by fluorimetry at $485 \mathrm{~nm}$ (excitation) and $538 \mathrm{~nm}$ (emission), respectively (Fluoroscan Ascent FL, Thermo Fisher Scientific). As transport of LY occurs exclusively by extracellular diffusion, this compound was also used as a control for the degree of paracellular passage through the Caco-2 layer. Potentially induced cytotoxicity was estimated through the release of intracellular lactate dehydrogenase into the extracellular medium. Activity of dehydrogenase was assayed by a cytotoxicity detection kit from Roche Diagnostics and compared with appropriate negative (absence of compound or condition to be tested) and positive (total cell lysis induced by $1 \%$ Triton, Sigma-Aldrich) controls.

\section{Experimental design}

Quantitative characterisation of selenium transport and influence of selenium concentration. Se was added to the afore-described cell culture system as a solution of individual Se species standards (Se(VI), Se(IV), SeMet and MeSeCys; Sigma-Aldrich) diluted in Hank's buffered salt solution (HBSS, Lonza) over a concentration range (25-50-100 and $200 \mathrm{ng} \mathrm{Se} / \mathrm{ml}$ ) ( $n$ 3). Before addition of Se, cells were systematically pre-incubated for $20 \mathrm{~min}$ in Se-free HBSS. For each treatment/replicate, a volume of $1.5 \mathrm{ml}$ of the respective Se standard was added to the AP chamber, and $2.5 \mathrm{ml}$ of HBSS containing $1 \%(\mathrm{w} / \mathrm{v})$ bovine serum albumin (Sigma-Aldrich) was added to the corresponding BL chamber. Cells were maintained in these conditions at $37^{\circ} \mathrm{C}$ for $3 \mathrm{~h}$, a plausible duration for the passage of food through the small intestine. At the end of the $3 \mathrm{~h}$ period, both AP and BL culture media were directly collected in clear glass vials and appropriately diluted for Se quantification. Cells were doubly washed with Se-free PBS solution (PBS) and scraped in $1 \mathrm{ml}$ of this solution. Subsequently, one-tenth of this quantity was used for protein determination via the Bicinchoninic Acid kit for Protein Determination (Sigma-Aldrich) and the rest was used for determination of cellular Se content. Cells in PBS were centrifuged at $13000 \mathrm{rpm}$ for $4 \mathrm{~min}$, the supernatant was removed and an enzymatic extraction was applied to the pellet in order to liberate Se. Extraction was carried out with protease (protease XIV, Sigma) in Tris- $\mathrm{HCl}$ buffer ( $30 \mathrm{~mm}$; Sigma-Aldrich) at $\mathrm{pH}$ 7.5 and $37^{\circ} \mathrm{C}$, with application of an ultrasonic probe (Vibra Cell, Bioblock Scientific) for $1.5 \mathrm{~min}$ per sample. Certified reference materials (SELM-1 (selenised yeast) NRCC; BCR185-R (beef muscle), IRMM and NIST-2976 (mussel tissue), NIST) were used to verify the efficacy of the extraction method. The preservation of Se species during extraction was verified by spiking experiments on SELM-1.

Methods for Se addition and sample collection were identical for all experiments in the present study and only specific differences in procedure for each experiment are further detailed.

Polarisation of selenium transport. Solutions of individual Se standards containing $100 \mathrm{ng} \mathrm{Se} / \mathrm{ml}$ were added either to the $\mathrm{AP}$ or the $\mathrm{BL}$ compartment, and Se species were quantified in the opposite compartment after $3 \mathrm{~h}$. LY and radiolabelled $\left[{ }^{14} \mathrm{C}\right]$-mannitol (ARC) were used as controls for paracellular passage. Mannitol was quantified using a liquid scintillation analyser (Packard 1600 TR; Perkin-Elmer) upon addition of scintillation solution (Pico-Fluor-15, Perkin-Elmer).

Influence of tight junction opening on selenium transport. Solutions of Se standards $(100 \mathrm{ng} \mathrm{Se} / \mathrm{ml})$ were added to the AP compartment for $3 \mathrm{~h}$. The opening of epithelial tight 
junctions was achieved by depriving them of $\mathrm{Ca}$ through the addition of $2.5 \mathrm{~mm}$-ethylene glycol tetraacetic acid (EGTA; Sigma-Aldrich), a chelator of $\mathrm{Ca}$ ions, to both $\mathrm{AP}$ and $\mathrm{BL}$ compartments.

Kinetics of selenium transport. Solutions of Se species (100 ng Se/ml) were added to the AP compartment, and aliquots from $\mathrm{AP}$ and $\mathrm{BL}$ media were sampled at six different time periods after Se addition (6-15-45-90-135-180 min). Volumes of collected aliquots corresponded to $5 \%$ of each chamber's volume, which represent $75 \mu \mathrm{l}$ in the AP chamber and $125 \mu \mathrm{l}$ in the BL chamber. Calculations of concentrations were adapted to remaining volumes. In this way, a constant ratio between the $\mathrm{AP}$ and $\mathrm{BL}$ volumes was maintained, preventing any effect of changes in solute concentration on Se transport.

Temperature dependence of selenium transport. Solutions of Se standards (100 ng Se/ml) were added to the AP compartment, and transport took place at either $37^{\circ} \mathrm{C}$ or at $4^{\circ} \mathrm{C}$. For the $4^{\circ} \mathrm{C}$ experiments, cells and solutions were pre-incubated for $20 \mathrm{~min}$ at $4^{\circ} \mathrm{C}$ in order to reduce enzymatic activity and to rigidify lipid plasma membranes, thus inactivating both active and passive carriers during Se transport. LY was added together with $\mathrm{Se}$ in order to compare the extent of paracellular transport at 37 and $4^{\circ} \mathrm{C}$. In contrast to other experiments, the duration of transport was for $2 \mathrm{~h}$ instead of $3 \mathrm{~h}$ in order to limit cold-induced cell damage.

Competition between selenium and sulphur analogues during transport. Caco-2 cells were treated with solutions containing Se species (100 $\mathrm{ng} \mathrm{Se} / \mathrm{ml}$ ) in the presence or absence of their respective $S$ analogues. The latter were added at concentrations 100 -fold higher $(10 \mu \mathrm{g} \mathrm{S} / \mathrm{ml})$ than the Se analogues. Respective analogues of Se(VI), Se(IV), MeSeCys and SeMet were sulphate, sulphite, methylcysteine and methionine (all from Sigma-Aldrich). These compounds have identical molecular structures, but differ only by the presence of an S atom replacing an Se atom in the same position.

\section{Chemical analysis of selenium}

Se species were quantified in AP and BL culture media, as well as in scraped cells. Se content of major medium components (DMEM, HBSS and FBS) was also determined. Speciation analyses were made with HPLC (Pro Star, Varian) coupled to inductively coupled plasma-MS (820-MS, Varian). The limit of quantification and the limit of detection were calculated as, respectively, six times and three times the baseline standard deviation, and the limit of quantification was $0.5 \mathrm{ng}$ $\mathrm{Se} / \mathrm{ml}$ for all Se species. Chromatographic separation of Se species was obtained with a reverse-phase C8 Alltima column $(250 \mathrm{~mm} \times 4.6 \mathrm{~mm}$ inner diameter $\times 5 \mu \mathrm{m}$ particle size; Grace Davison Discovery Science) using a mobile phase consisting of $2 \%(\mathrm{v} / \mathrm{v})$ heptafluorobutyric acid (Alfa Aesar) in a gradient with $50 \%(\mathrm{v} / \mathrm{v})$ methanol (HPLC grade; VWR). Total elution of all Se species was obtained within $10 \mathrm{~min}$ at a flow rate of $1 \mathrm{ml} / \mathrm{min}$. The isotope ${ }^{78} \mathrm{Se}$ was used for measurement with $90 \mathrm{ml} / \mathrm{min}$ of $\mathrm{H}_{2}$ as reaction gas to remove spectral polyatomic interferences on this mass. All dilutions were made with milliQ water (Millipore).
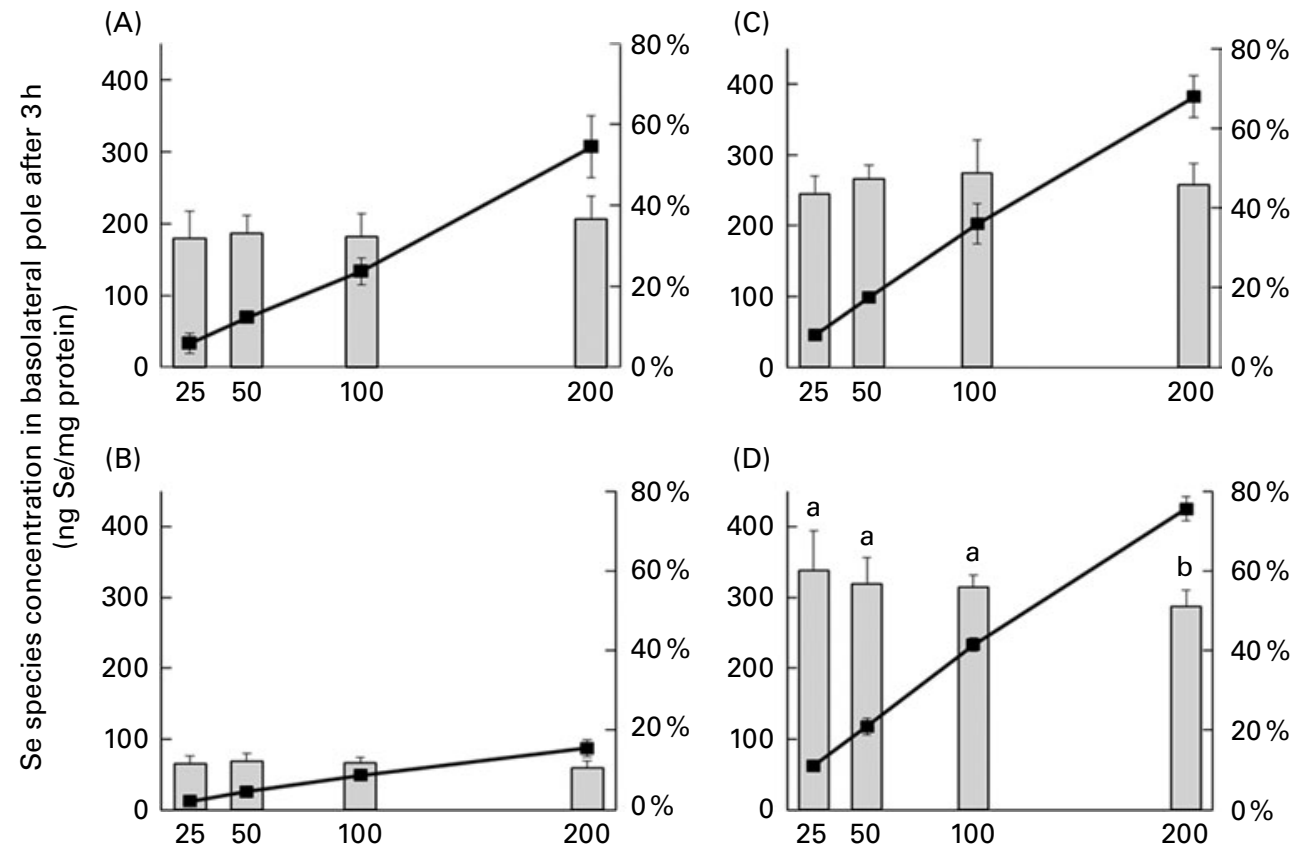

(D)

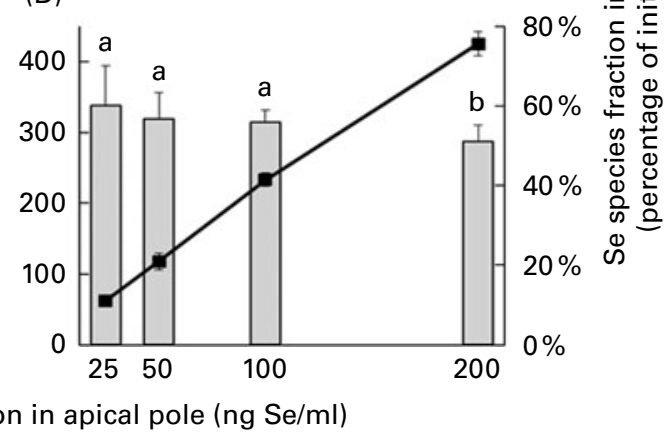

Fig. 1. Fractions and concentrations of $(A)$ selenate, $(B)$ selenite, $(C)$ methylselenocysteine and (D) selenomethionine in the basolateral compartment at $3 \mathrm{~h}$ after having been added to the apical compartment as individual standards at various concentrations (25, 50,100 and $200 \mathrm{ng}$ selenium/ml). Lines show results expressed as ng selenium/mg protein and columns show percentages of initial added selenium. Values are means, with standard deviations represented by vertical bars. For a given species, all selenium values expressed in ng selenium/mg protein were significantly different from each other $(P \leq 0.5)$. ${ }^{a, b}$ Mean values with unlike letters were significantly different for selenium values expressed in percentage $(P \leq 0.5)$. 


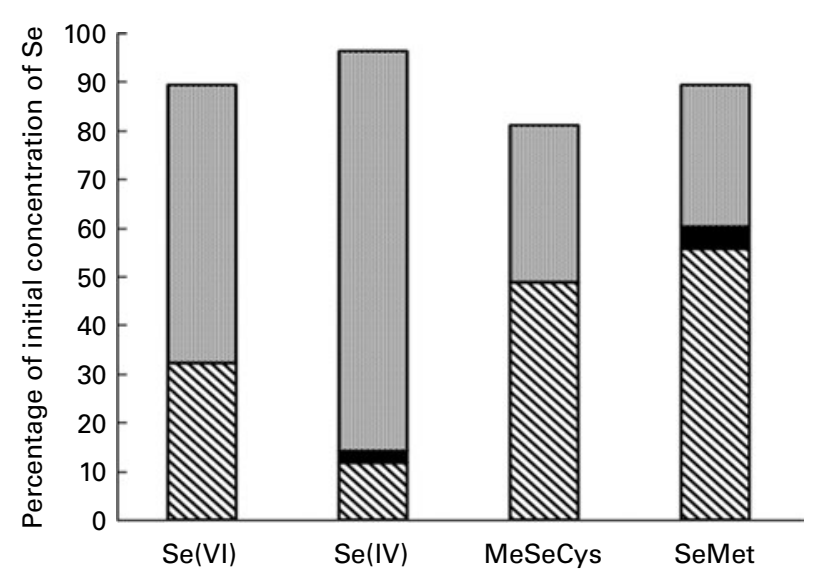

Fig. 2. Distribution of selenate (Se(VI)), selenite (Se(IV)), methylselenocysteine (MeSeCys) and selenomethionine (SeMet) in different locations of the cell culture system at $3 \mathrm{~h}$ after having been added to the apical compartment

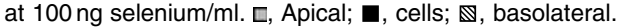

\section{Statistical analysis}

Results are given as mean values and standard deviations. Each experiment was repeated three times $(n 3)$ and samples were produced in duplicate for each repetition $\left(\begin{array}{ll}n & 2) \text {. The }\end{array}\right.$ effect of concentration on Se absorption was analysed by one-way ANOVA, followed by Tukey's honestly significant difference multiple comparison procedure in order to compare means of individual species. The effects of temperature and of the presence of EGTA and S compounds were analysed by a Student's $t$ test in comparison to control conditions, i.e. at $37^{\circ} \mathrm{C}$ and without EGTA or S. Software used was JMP 9.0.0 for Windows (SAS Institute). Differences with a $P$ value less than or equal to 0.05 were considered as being statistically significant ( $95 \%$ confidence level).

\section{Results}

\section{Monitoring of cell culture system}

Potential cytotoxicity was found to be less than $10 \%$ of the positive control for all experimental conditions. TEER values prior to the start of the experiment ranged from 1100 to $1400 \Omega \mathrm{cm}^{2}$, which ensured the integrity of the cell monolayer $^{(20)}$. A decrease of about $10 \%$ of initial TEER values was systematically observed at the end of the experiment (except in the presence of EGTA, where there was a greater decrease). Average protein content of cells was 0.26 (SD 0.08) $\mathrm{mg}$ per insert, corresponding to $56.7 \mu \mathrm{g} / \mathrm{cm}^{2}$. No Se was detected in either DMEM or HBSS, but a concentration of $20 \mathrm{ng} \mathrm{Se} / \mathrm{ml}$ was measured in pure FBS, mainly in the form of Se(IV) and SeMet. This means that $2 \mathrm{ng} / \mathrm{ml}$ of Se was present in the $10 \%$ FBS nutritive medium. When $0.5 \%$ of FBS was used during the last $2 \mathrm{~d}$ of culture, Se background in media and cells was reduced to a value below the limit of detection. Transport of LY was similar (within 1 and $2 \%$ of initial LY) in the presence or absence of Se compounds, with no difference between Se species, which indicated that Se had no effect on tight junctions opening.

\section{Experimental results}

Quantitative characterisation of selenium transport and influence of selenium concentration. For all Se species and at all concentrations applied, Se species recovered in the BL compartment were identical to those that were initially added to the AP compartment, clearly indicating that no transformation of species occurred during transport. Within the applied concentration range $(25-200 \mathrm{ng} \mathrm{Se} / \mathrm{ml})$, the degree of transport of Se(VI), Se(IV) and MeSeCys from the AP to BL compartment was proportional to the concentration applied (Fig. 1). Indeed, whatever the initial AP Se concentration, a constant fraction of Se (as a percentage of initial Se) was recovered in the BL compartment. In contrast, SeMet was recovered in a smaller proportion in the BL compartment when added at a concentration of $200 \mathrm{ng} \mathrm{Se} / \mathrm{ml}$, compared with the lower initial concentrations. In general, efficiency of Se absorption was as follows: Se(IV) (12 (SD 1)\%) < Se(VI) (33 (SD 2)\%) $<$ MeSeCys (46 (SD 2)\%) < SeMet (56 (SD 4)\%). Fig. 2 shows the distribution of Se species in different locations of the cell culture system, $3 \mathrm{~h}$ after the addition of Se species. Proteolytic extraction of cells revealed the presence of detectable amounts of Se following enrichment with both Se(IV) and SeMet. Intracellular Se(IV) and SeMet accounted, respectively, for 2 and $4 \%$ of initial Se.

Polarisation of selenium transport. To allow for a better comparison between data obtained for Se, LY and mannitol, results of the present experiment were expressed as clearance values. These correspond to the volume of solution containing the compound of interest that passed across the cell monolayer per $\mathrm{h}(\mu \mathrm{l} / \mathrm{h})^{(21)}$. Data presented in Table 1 show that the BL to AP passage was low and very similar for all Se species and control substances (LY and mannitol). This suggests that all were transported at the same low level, most probably by the paracellular route. Both LY and mannitol controls showed clearance ratios (i.e. clearance value in the $\mathrm{AP}-\mathrm{BL}$ way compared with the same value for the $\mathrm{BL}-\mathrm{AP}$ way) close to 1 , which indicates a non-polarised transport. Among Se species, Se(IV) also had a clearance ratio close to 1 (1.9), but the three other Se species showed considerably higher ratios, which means that their transport across the

Table 1. Clearance values and ratios for selenium species (100 ng selenium $/ \mathrm{ml}$ ) in comparison with lucifer yellow (LY) and mannitol, from the apical (AP) to the basolateral (BL) pole and inversely, $3 \mathrm{~h}$ after selenium addition

(Mean values and standard deviations)

\begin{tabular}{|c|c|c|c|c|c|}
\hline & \multicolumn{5}{|c|}{ Clearance } \\
\hline & \multicolumn{2}{|c|}{ AP-BL $(\mu \mathrm{l} / \mathrm{h})$} & \multicolumn{2}{|c|}{ BL-AP $(\mu \mathrm{l} / \mathrm{h})$} & \multirow{2}{*}{$\begin{array}{l}\text { Clearance }_{\mathrm{AP}-\mathrm{BL}} \\
\text { clearance }_{\mathrm{BL}-\mathrm{AP}}\end{array}$} \\
\hline & Mean & SD & Mean & $\mathrm{SD}$ & \\
\hline $\mathrm{Se}(\mathrm{VI})$ & 101.4 & 24.9 & $14 \cdot 0$ & $4 \cdot 3$ & $7 \cdot 3$ \\
\hline Se(IV) & $28 \cdot 6$ & $15 \cdot 0$ & $15 \cdot 2$ & 5.9 & 1.9 \\
\hline SeMet & 233.9 & 34.6 & $12 \cdot 5$ & 1.9 & $18 \cdot 7$ \\
\hline MeSeCys & $186 \cdot 4$ & $6 \cdot 4$ & $13 \cdot 9$ & 2.7 & $13 \cdot 5$ \\
\hline Mannitol & $13 \cdot 6$ & 1.7 & $12 \cdot 9$ & 0.2 & $1 \cdot 1$ \\
\hline LY & $9 \cdot 3$ & 2.7 & $9 \cdot 2$ & 0.8 & $1 \cdot 0$ \\
\hline
\end{tabular}

$\mathrm{Se}(\mathrm{VI})$, selenate; Se(IV), selenite; SeMet, selenomethionine; MeSeCys, methylselenocysteine. 


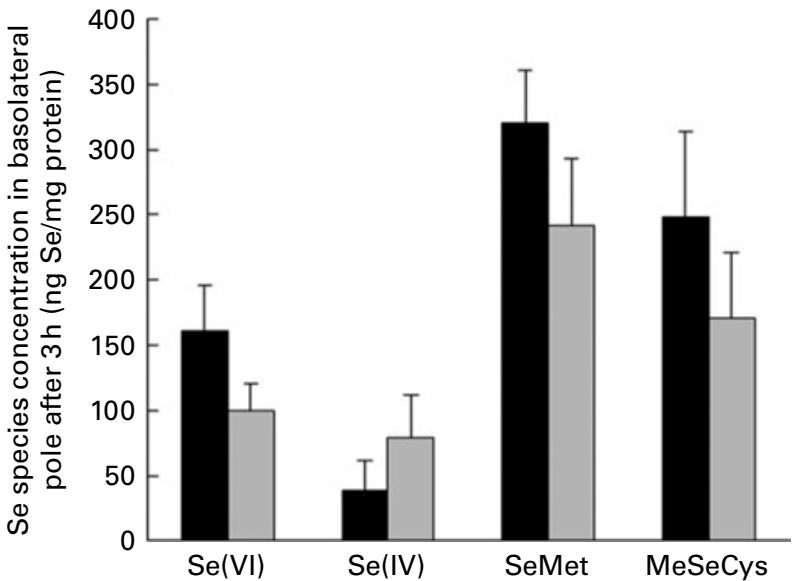

Fig. 3. Concentrations of selenate $(\mathrm{Se}(\mathrm{VI}))$, selenite (Se(IV)), methylselenocysteine (MeSeCys) and selenomethionine (SeMet), per $\mathrm{mg}$ of protein, in the basolateral compartment at $3 \mathrm{~h}$ after having been added to the apical compartment at $100 \mathrm{ng}$ selenium $/ \mathrm{ml}$ in the presence or absence of EGTA. Values are means, with standard deviations represented by vertical bars. For each species, results in the presence or absence of EGTA were significantly different from each other $(P \leq 0.05)$. $\mathbf{n}$, Selenium; $\square$, selenium + EGTA.

cell monolayer was importantly polarised in the AP-BL way. The accumulation of SeMet and Se(IV) in enterocytes was also polarised, as no intracellular Se was detected following BL addition.

Influence of tight junction opening on selenium transport. The effect of EGTA on relaxing tight junctions and the concurrent increase in paracellular diffusion were verified after the $3 \mathrm{~h}$ treatment by TEER values that reduced to $35 \%$ of their initial resistance and by a six-fold increase in LY clearance (from 9 to $54 \mu \mathrm{l} / \mathrm{h}$ ). Se(IV) was the only species whose transport was amplified by EGTA (2-2-fold increase) (Fig. 3). All the other species had their passage lowered in the presence of EGTA.

Kinetics of selenium transport. Recovery of Se in the BL chamber always increased with the duration of exposure, regardless of the species that was added to the AP compartment. However, organic and inorganic Se species showed distinct absorption profiles (Fig. 4)

(1) Transport of SeMet and MeSeCys started very quickly after addition of Se to the AP compartment. After 15 min, Se concentration in the BL chamber was greater than $50 \%$ of the Se concentration measured after $3 \mathrm{~h}$. In contrast, inorganic Se species were only detected in the BL chamber 45 min after the start of exposure.

(2) While concentrations of Se(IV) and Se(VI) in the BL compartments were still increasing at the end of the $3 \mathrm{~h}$ experiment, absorption curves of SeMet and MeSeCys reached a plateau, suggesting that their transport was almost at its maximum. At that moment, final concentrations (per volume unit) were almost equal in both compartments for SeMet and MeSeCys. In contrast, final BL concentrations of the inorganic species were well below AP concentrations.

Temperature dependence of selenium transport. Se(IV) was the only species whose transport was not affected by low temperature, similar to LY (Fig. 5). At $4^{\circ} \mathrm{C}$, there was no significant difference between final BL concentrations of Se species. In addition, the clearance values at this temperature were very close to those found for LY. It is probable that, at $4^{\circ} \mathrm{C}$, Se transport was only composed of the paracellular fraction.

Competition for transport between selenium and sulphur analogues. Fig. 6 shows that transport of SeMet and MeSeCys was significantly reduced in the presence of their respective S-analogues. The observed reductions were 62 and $60 \%$, respectively. Transport of inorganic Se species did not significantly decrease in the presence of sulphate or sulphite.

\section{Discussion}

\section{Cell culture system}

Se bioavailability has been investigated using animal ${ }^{(14-16,22,23)}$ and human ${ }^{(17,18,24)}$ models, in which bioavailability was estimated by determination of Se content in blood or in tissues after ingestion of specific Se species. Compared to in vivo models, recent in vitro cell culture systems allow more rapid and easy variations of some relevant parameters such as Se concentration, absorption duration, temperature or addition of certain components, which may help to obtain a deeper insight into mechanistic processes at the absorptive level. Caco-2 cells used in the present in vitro model originate from a human colon adenocarcinoma and express, upon full differentiation, several morphological and biochemical characteristics of intestinal absorptive cells (enterocytes), such as cell polarisation, presence of microvilli and tight junctions or expression of membranous transporters ${ }^{(25,26)}$. In practice, cells were cultivated on bicameral permeable inserts placed

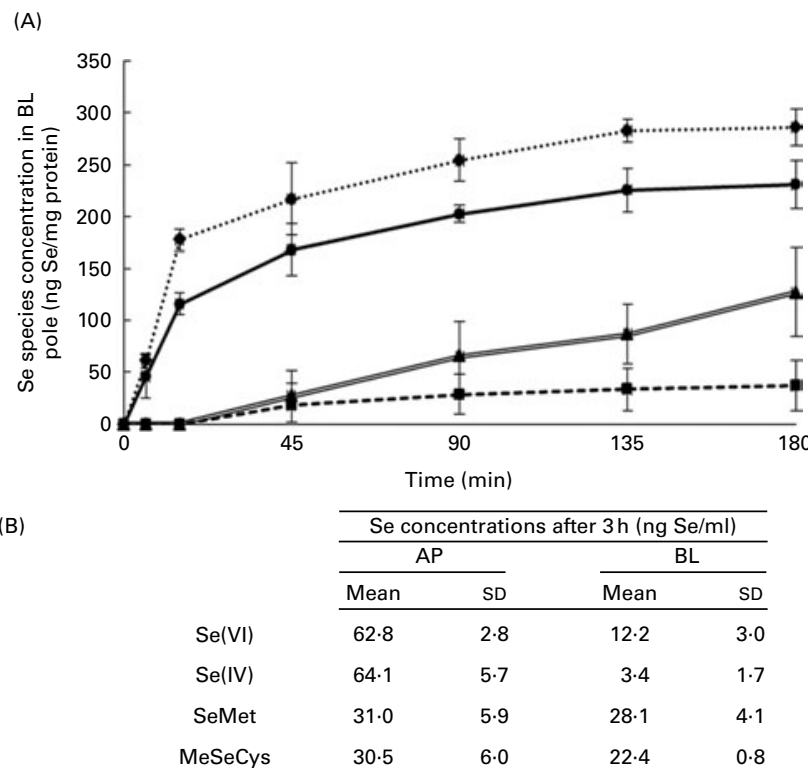

Fig. 4. (A) Concentrations of selenate ( $\mathrm{Se}(\mathrm{VI}), \boldsymbol{\sim})$, selenite (Se(IV), - - methylselenocysteine (MeSeCys, - - ) and selenomethionine (SeMet, - - ), per $\mathrm{mg}$ of protein, in the basolateral (BL) compartment at $6,15,45,90,135$ and $180 \mathrm{~min}$ after having been added to the apical (AP) compartment (100 ng selenium $/ \mathrm{ml}$ ). (B) Corresponding concentrations of selenium species per volume unit in the AP and BL poles after $180 \mathrm{~min}$. Values are means, with standard deviations represented by vertical bars. 


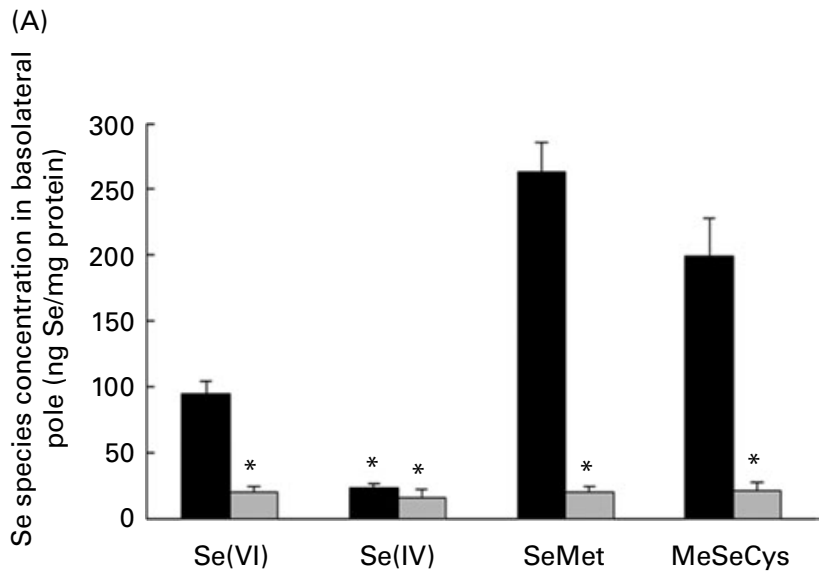

(B)

\begin{tabular}{lrrrrr}
\cline { 2 - 3 } & \multicolumn{4}{c}{ Clearance $(\mu \mathrm{l} / \mathrm{h})$} \\
\cline { 2 - 3 } \cline { 5 - 6 } Se(VI) & 95.2 & 8.9 & & 19.7 & 4.1 \\
Se(IV) & 22.7 & 4.1 & & 16.0 & 5.6 \\
SeMet & 263.3 & 22.4 & 20.1 & 4.7 \\
MeSeCys & 198.9 & 29.6 & & 21.1 & 6.6 \\
$\quad$ Ly & 11.1 & 3.4 & 10.5 & 2.6
\end{tabular}

Fig. 5. (A) Concentrations of selenate (Se(VI)), selenite (Se(IV)), methylselenocysteine (MeSeCys) and selenomethionine (SeMet), per $\mathrm{mg}$ of protein, in the basolateral compartment at $2 \mathrm{~h}$ after having been added to the apical compartment at $100 \mathrm{ng}$ selenium/ml. Values are means, with standard deviations represented by vertical bars. * Mean values are not significantly different from each other. (B) Corresponding clearance values for selenium species and lucifer yellow. $\square, 37^{\circ} \mathrm{C} ; \square, 4^{\circ} \mathrm{C}$.

in culture wells in which the upper (AP) chamber mimicked the intestinal lumen while the lower $(\mathrm{BL})$ chamber mimicked the bloodstream. Albumin, the principal plasma Se transporter $^{(27)}$, was added to the BL compartment to simulate blood and extravascular fluids. The enrichment concentration of $100 \mathrm{ng} \mathrm{Se} / \mathrm{g}$, which was used for most experiments, corresponded to a dose slightly higher than the usual Se intake that would have been diluted in 1 litre of drinking water and digestive fluids. This concentration allowed analytically detectable responses at early collection time points of the kinetics experiment and in the presence of S-containing Se analogues. The inevitable presence of Se in serum could potentially lead to Se accumulation in cells, and could thereby affect transcellular transport by limiting the entry of compounds inside the cells. For this reason, the FBS fraction in the medium was reduced during the last $2 \mathrm{~d}$ of culture, and transport experiments were conducted in Se-free media. This allowed us to obtain cells virtually free from Se (less than limit of detection). In addition, Se concentrations in enrichment solutions were in excess of Se concentration in the medium, which also prevented a substantial influence of endogenous $\mathrm{Se}$ on the exogenous species transport.

\section{Efficiency of selenium species transport}

Se transport across the Caco-2 monolayer was clearly influenced by speciation. Organic species showed greater absorption than inorganic species over the $3 \mathrm{~h}$ experimental exposure period. The extrapolation of the present cellular model to the human intestinal tract would indicate that $56 \%$ of the SeMet released from the food matrix into the small intestine is absorbable through the intestinal barrier for potential further metabolic utilisation, i.e. it is bioavailable. Correspondingly, bioavailable fractions of MeSeCys, Se(VI) and Se(IV) are, respectively, 46, 33 and $12 \%$. In addition, Caco-2 cells were shown to accumulate significant quantities of Se(IV) and SeMet, exclusively from the AP pole. Neither $\mathrm{Se}(\mathrm{VI})$ nor MeSeCys were detected in cells. In a previous study, Caco-2 cells were reported to accumulate Se from MeSeCys to a similar extent to Se from Se(IV) ${ }^{(28)}$. Considering that the duration of these experiments were $72 \mathrm{~h}$ in the reported study, $v .3 \mathrm{~h}$ in the present study, it is possible that Se(IV) accumulates more rapidly in cells compared with MeSeCys. Intracellular SeMet content detected during the present study was high compared with that of Se(IV). However, on addition of high concentrations of Met, no SeMet was detected in cells. This illustrates the inability of cellular Mettransfer RNA to discriminate between SeMet and Met. In the presence of SeMet, translational mechanisms permit the integration of SeMet instead of Met in order to generate socalled 'Se-containing proteins'. SeMet can accumulate in cells in substantial quantities as long as Se supply is sufficient ${ }^{(29)}$. This ability of SeMet to be stored in body proteins represents an interesting mechanism, not shared by other Se species that have much shorter half-lives in the body ${ }^{(30)}$.

\section{Characterisation of selenium species transport}

Se species transported via a transcellular route might undergo metabolic transformations inside cells prior to their release to the BL side. In previous in vitro studies, bioavailable Se was systematically reported as total Se without reference to speciation $^{(28,31)}$. In the present study, speciation analysis was performed by HPLC-inductively coupled plasma-MS on the products of the transport experiments. This showed that Se species remained in their original form after crossing the

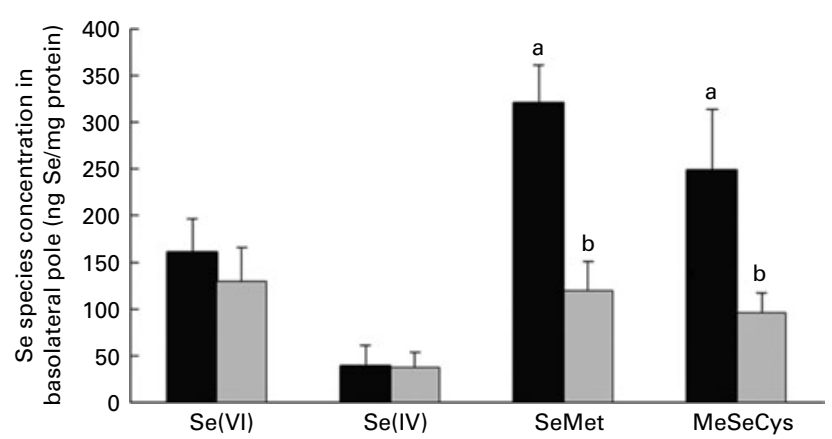

Fig. 6. Concentrations of selenate (Se(VI)), selenite (Se(IV)), methylselenocysteine (MeSeCys) and selenomethionine (SeMet), per mg of protein, in the basolateral compartment at $3 \mathrm{~h}$ after having been added to the apical compartment $(100 \mathrm{ng}$ selenium $/ \mathrm{ml})$ in the presence or absence of their sulphur analogue $(10 \mu \mathrm{g}$ selenium $/ \mathrm{ml})$. Values are means, with standard deviations represented by vertical bars. ${ }^{a, b}$ Mean values with unlike letters are significantly different from each other for a given species $(P \leq 0.05)$. $\mathbf{n}$, Selenium; $\square$, selenium + sulphur. 
Caco-2 monolayer. Hence, Se species seem to cross the intestinal barrier without metabolic transformation.

Transport across the intestinal epithelium can be achieved by paracellular or transcellular pathways, with both modes of transport making variable contributions to the total Se transported. During paracellular transport, nutrients simply diffuse through extracellular spaces along a concentration gradient. Because of numerous tight junctions that bind epithelial cells closely together, paracellular passage is limited to hydrophilic molecules of low molecular weight and low hydrodynamic dimension, which is the case of Se species under investigation here. On the other hand, when using a more efficient transcellular pathway, nutrients enter the cells during their transport process. As the phospholipid cell plasma membrane restricts the diffusion of hydrophilic Se compounds, passive or active membrane transporters are expected to be required for transcellular passage of $\mathrm{Se}^{(32,33)}$.

In the present study, identification of the para- or transcellular route was primarily based on the degree of polarisation of Se species transport. A clearance ratio of 1 corresponds to a non-polarised transport and reveals that a compound should be exclusively using the paracellular route. Clearance ratios $>1$ relate to compounds having an additional transcellular transport component whose importance is proportional to the size of this ratio. The use of EGTA was also expected to reveal the occurrence of para- or transcellular transport as this chelating agent has a particular affinity for $\mathrm{Ca}$, which is necessary to ensure epithelial tight junction integrity. The presence of EGTA entails a decrease in free $\mathrm{Ca}^{2+}$ that causes loosening of the junctions and increases paracellular diffusion $^{(32)}$. As a consequence, in the presence of EGTA, Se species using the paracellular route should show enhanced transport in comparison with Se species transported transcellularly. Results obtained in the present study were surprising, in the sense that three of the four Se species had their transport lowered by EGTA rather than unchanged or slightly increased, as was expected considering that their paracellular fraction of transport had been amplified. An explanation could be the existence of a Ca-dependent transport process, which would have been inhibited by EGTA but, to our knowledge, such a mode of transport has never been reported for Se. Despite a lack of explanation for this decrease in Se transport, we could identify two distinct forms of behaviour for Se species in combination with EGTA: either an increase or a decrease in Se passage. We propose that an EGTA-mediated increase in Se mobility should be indicative of a paracellular transport pathway, while a decrease suggests predominantly transcellular transport.

A second step in Se transport characterisation was the detection of the action of membrane-bound transporters, achieved by establishing kinetic profiles of Se species absorption. Carrier-mediated transport has typical enzyme-like kinetics, i.e. rapid increasing absorption followed by a saturation plateau corresponding to a full occupation of transporters working at their maximal velocity $\left(V_{\max }\right)$. In contrast, paracellular diffusion has a profile of absorption that is time proportional and of low importance with saturation occurring after a much longer period, i.e. when concentrations are equal in both compartments. The occurrence of transporters was also assessed by an investigation into the temperature dependence of Se mobility. At low temperature, most cellular enzymes are inhibited, which considerably slows down catabolic reactions providing ATP and inactivates energy-dependent transporters. In addition, at $4^{\circ} \mathrm{C}$, the plasma membrane becomes significantly more rigid so that membranous ion channels are expected to contract and become blocked or highly restricted. In this way, at $4^{\circ} \mathrm{C}$, only non-carrier-mediated transport is maintained.

Finally, concerning the identity of potential Se transporters, it is probable that mechanisms involved in $\mathrm{S}$ transport also play a role in the transport of Se-compounds due to important chemical and morphological similarities between Se species and their $\mathrm{S}$ analogues ${ }^{(34)}$. The existence of common transporters is expected to generate competition for absorption between Se and S, which is affected by the affinities of the transporters for one or the other type of compound.

\section{Selenate}

In a previous in vitro study, Se(VI) was proposed to be transported by the paracellular route because a non-polarised transport had been observed (apparent permeability $\left(P_{\text {app }}\right)$ ratio, i.e. parameter comparable to the clearance ratio, close to 1$)^{(31)}$. However, the present results do not support this hypothesis. First, the clearance ratio was largely superior to 1 (7.3). Moreover, the presence of EGTA did not favour Se(VI) passage. Third, despite the typically limited extent of paracellular diffusion, the efficiency of Se(VI) transport reached $33 \%$. Se(VI) passage was reduced to its paracellular fraction at low temperature, which suggests a role for membrane transporters as they become inhibited in these conditions. We could not observe any significant competition between Se(VI) and sulphate, despite a sulphate concentration 100-fold higher than Se(VI). A common transport mode for sulphate and Se(VI) can, however, not be completely excluded, because a slight inhibition was noticed and because no saturation in Se(VI) transport was observed after $3 \mathrm{~h}$. We can therefore hypothesise that, in the present experimental conditions, Se(VI)/sulphate transporters were not saturated and allowed passage of both compounds. This hypothesis would support the conclusions of Wolffram et al. ${ }^{(35)}$, who identified, based on experiments conducted on brushborder membrane of pig jejunum, two active modes of transport $\left(\mathrm{Na}^{+}\right.$- and $\mathrm{H}^{+}$-co-transports) used by both sulphate and Se(VI).

\section{Selenite}

Se(IV) had the lowest clearance ratio among Se species, with a value of 1.9 . This suggests that Se(IV) transport occurred mainly via a paracellular route. The absence of an effect of low temperature and of the presence of sulphite, in combination with an increased transport in the presence of EGTA and a low and progressive absorption profile, were also all in favour of a paracellular mode of transport. These observations are in accordance with the conclusions of a previous 
study suggesting a passive diffusion of Se(IV) based on a $P_{\text {app }}$ ratio of approximately $1^{(31)}$.

\section{Selenomethionine and methylselenocysteine}

SeMet and MeSeCys behaved rather similarly under the present experimental conditions. Their AP to BL transport was highly polarised, suggesting a dominant transcellular transport mode. In addition, SeMet and MeSeCys transport strongly decreased at low temperature and was partially inhibited by their respective S-analogues. The present results thus indicate that SeMet and MeSeCys are transported, at least to a certain extent, by an AP transport system shared with their S-analogue. Whereas there is little published data on MeSeCys absorption, the existence of a common transporter for SeMet and Met is better documented ${ }^{(31,36)}$. Two active mechanisms of SeMet transport were identified in differentiated Caco-2 cells, one of them being $\mathrm{Na}^{+}$dependent ${ }^{(37)}$. These transporters also were reported to have similar affinity for Se- and Scompounds $^{(38)}$. In the present experiment, inhibition of Se transport by $\mathrm{S}$ compounds was not complete, although Met and methylcysteine concentrations were 100-fold higher than those of the corresponding Se compounds, which suggests the existence of additional transport mechanisms.

SeMet and MeSeCys both exhibited saturation kinetics of absorption following their addition at $100 \mathrm{ng} / \mathrm{ml}$ to the AP compartment. Saturation was concomitant with the diminution of the concentration gradient existing between the $\mathrm{AP}$ and $\mathrm{BL}$ chambers. Velocity of transport depended on the importance of the initial concentration gradient applied to the cell culture system. Indeed, higher final Se concentrations were measured in the BL pole after initial Se concentrations of $200 \mathrm{ng} / \mathrm{ml} \mathrm{com-}$ pared to $100 \mathrm{ng} / \mathrm{ml}$, demonstrating a more rapid transport associated with the highest initial concentration of Se. Final BL concentration of MeSeCys was exactly two times more important when initial concentration was doubled. In the case of SeMet, final BL concentration was slightly lower than the exact double, meaning that maximal velocity $\left(V_{\max }\right)$ of SeMet transporters was closer to being reached compared to MeSeCys. The $V_{\max }$ values of Se transport thus seem to be species dependent, with SeMet transporters reaching their $V_{\max }$ at slightly lower Se concentrations in comparison with MeSeCys transporters.

\section{Conclusion}

The existence of a species-dependent degree of bioavailability of Se is well-known. In the present study, bioavailability of Se could be related to differences in efficiency of Se species absorption at the intestinal level. An explanation for these differences in Se absorption is the fact that Se species follow distinct transport routes through the intestinal barrier. Se(IV) crosses the intestinal epithelium almost exclusively by a passive paracellular pathway. In contrast, Se(VI) transport is for a great part transcellular, with a possible intervention of the sulphate transporter. Both SeMet and MeSeCys follow transcellular pathways and are able to use the same AP transporters as their S-analogues. In addition, SeMet can be stored in cellular proteins, which represents an Se supply in case of shortage. In line with their modes of transport, Se(IV) turns out to be poorly bioavailable, while SeMet is the most available Se species, followed by MeSeCys. The present results suggest that, in the context of Se diet enrichment, preference should be given to organic forms of Se compared to inorganic ones. Se(IV) seems to be of particularly low nutritional value, as most of it will be directly excreted.

\section{Acknowledgements}

The present work was supported by the 'Fond Special de Recherche' from the Catholic University of Louvain-la-Neuve (UCL) (in vitro experiments) and by the Belgian Federal Science Policy (optimisation of the Se speciation method). In vitro work was conducted at UCL and Se analysis was carried out at CODA-CERVA. C. T. and Y.-J. S. designed the research; C. T. conducted the research, analysed the data and wrote the manuscript; A. R. supported the redaction of the manuscript; Y.-J. S. and L. P. revised the final manuscript. All authors have read and approved the final paper. None of the authors has a conflict of interest to declare.

\section{References}

1. Schwarz K \& Foltz CM (1957) Selenium as an integral part of factor-3 against dietary necrotic liver degenaration. $J \mathrm{Am}$ Chem Soc 79, 3292-3293.

2. Gromer S, Eubel BL, Lee BL, et al. (2005) Human selenoproteins at a glance. Cell Mol Life Sci 62, 2414-2437.

3. Tapiero H, Townsend DM \& Tew KD (2003) The antioxidant role of selenium and seleno-compounds. Biomed Pharmacother 57, 134-144.

4. Sengupta A, Lichti UF, Carlson BA, et al. (2010) Selenoproteins are essential for proper keratinocyte function and skin development. PLoS One 5, e12249.

5. Navarro-Alarcón M \& López-Martínez MC (2000) Essentiality of selenium in the human body: relationship with different diseases. Sci Total Environ 249, 347-371.

6. Clark LC, Combs GF, Turnbull BW, et al. (1996) Effects of selenium supplementation for cancer prevention in patients with carcinoma of the skin. A randomized controlled trial. Nutritional Prevention of Cancer Study Group. JAMA 276, 1957-1963.

7. Combs GF \& Gray WP (1998) Chemopreventive agents: selenium. Pharmacol Ther 79, 179-192.

8. Ip C, Thompson HJ, Zhu Z, et al. (2000) In vitro and in vivo studies of methylseleninic acid: evidence that a monomethylated selenium metabolite is critical for cancer chemoprevention. Cancer Res 60, 2882-2886.

9. Lederer J (1986) Sélenium et vitamine E (Selenium and vitamin E). Brussels: Nauwelaerts.

10. Fishbein L (1991) Selenium. In Metals and their Compounds in the Environment, pp. 1153-1190 [E Merian, editor]. Weinheim: VCH.

11. CSS (Conseil Supérieur de la Santé) (2009) Recommandations nutritionnelles pour la Belgique (Nutritional Recommendations for Belgium), no. 8309. Brussels: Superior Health Council.

12. Meltzer HM, Bibow K, Paulsen IT, et al. (1993) Different bioavailability in humans of wheat and fish selenium as 
measured by blood platelet response to increased dietary Se. Biol Trace Elem Res 36, 229-241.

13. Tiwary AK, Stegelmeier BL, Panter KE, et al. (2006) Comparative toxicosis of sodium selenite and selenomethionine in lambs. $J$ Vet Diagn Invest 18, 61-70.

14. Whanger PD \& Butler JA (1988) Effects of various dietary levels of selenium as selenite or selenomethionine on tissue selenium levels and glutathione peroxidase activity in rats. J Nutr 118, 846-852.

15. Wang Y, Han J, Li W, et al. (2007) Effect of different selenium source on growth performances, glutathione peroxidase activities, muscle composition and selenium concentration of allogynogenetic crucian carp (Carassius auratus Gibelio). Anim Feed Sci Technol 134, 243-251.

16. Wang Y \& Xu B (2008) Effect of different selenium source (sodium selenite and selenium yeast) on broiler chickens. Anim Feed Sci Technol 144, 306-314.

17. Thompson CD, Robinson MF, Campbell DR, et al. (1982) Effect of prolonged supplementation with daily supplements of selenomethionine and sodium selenite on gluthatione peroxidase activity in blood of New Zealand residents. $\mathrm{Am}$ J Clin Nutr 36, 24-31.

18. Griffiths NM, Stewart RDH \& Robinson MF (1976) The metabolism of [75Se]selenornethionine in four women. $\mathrm{Br} \mathrm{J}$ Nutr 35, 373-382.

19. Schümann K, Classen HG, Hages M, et al. (1997) Bioavailability of oral vitamins, minerals, and trace elements in perspective. Arzneimittelforschung 47, 369-380.

20. Biganzoli E, Cavenaghi LA, Rossi R, et al. (1999) Use of a Caco-2 cell culture model for the characterization of intestinal absorption of antibiotics. Farmaco 54, 594-599.

21. Berger V, De Bremaeker N, Larondelle Y, et al. (2000) Transport mechanisms of the imino acid L-proline in the human intestinal epithelial Caco-2 cell line. J Nutr 130, 2772-2779.

22. Wang C \& Lovell RT (1997) Organic selenium sources, selenomethionine and selenoyeast, have higher bioavailability than an inorganic selenium source, sodium selenite, in diets for channel catfish (Ictalurus punctatus). Aquaculture 152, 223-234.

23. Rider SA, Davies SJ, Jha AN, et al. (2010) Bioavailability of co-supplemented organic and inorganic zinc and selenium sources in a white fishmeal-based rainbouw trout (Oncorbynchus mykiss) diet. J Anim Physiol Anim Nutr 94, 99-110.

24. Levander OA (1983) Considerations in the design of selenium bioavailability studies. Fed Proc 42, 1721-1725.

25. Sambuy Y, De Angelis I, Ranaldi G, et al. (2005) The Caco-2 cell line as a model of the intestinal barrier: influence of cell and culture-related factors on Caco-2 cell functional characteristics. Cell Biol Toxicol 21, 1-26.

26. Hidalgo IJ \& Li J (1996) Carrier mediated transport and efflux mechanisms in Caco-2 cells. Adv Drug Deliv Rev 22, 53-66.

27. Suzuki Y, Hashiura Y, Matsumura K, et al. (2009) Dynamic pathways of selenium metabolism and excretion in mice under different selenium nutritional stresses. Metallomics $\mathbf{2}$, $126-132$.

28. Zeng H, Jackson MI, Cheng WH, et al. (2011) Chemical form of selenium affects its uptake, transport, and glutathione peroxidase activity in the human intestinal Caco-2 cell model. Biol Trace Elem Res 143, 1209-1218.

29. Swanson CA, Patterson BH, Levander OA, et al. (1991) Human $\left[{ }^{74}\right.$ Se]selenomethionine metabolism: a kinetic model. Am J Clin Nutr 54, 917-926.

30. Schrauzer GN (2000) Selenomethionine: a review of its biological significance, metabolism and toxicity. J Nutr 130, 1653-1656.

31. Knipp GT, Ho NF, Barsuhn CL, et al. (1997) Paracellular diffusion in Caco-2 cell monolayer: effect of perturbation on the transport of hydrophilic compounds that vary in charge and size. J Pharm Sci 86, 1105-1110.

32. Tang VW \& Goodenough DA (2003) Paracellular ion channel at the tight junction. Biophys J 84, 1660-1673.

33. Johansson L, Gafvelin G \& Arner ESJ (2005) Selenocysteine in proteins - properties and biotechnological use. Biochim Biophys Acta 1726, 1-13.

34. Leblondel G, Mauras Y, Cailleux A, et al. (2001) Transport measurements across Caco-2 monolayers of different organic and inorganic selenium. Biol Trace Elem Res $\mathbf{8 3}$, 191-206.

35. Wolffram S, Grenacher B \& Scharrer E (1988) Transport of selenate and sulfate across the intestinal brush-border membrane of pig jejunum by two common mechanisms. Q J Exp Physiol 73, 103-111.

36. Bakke AM, Tashjian DH, Wang CF, et al. (2010) Competition between selenomethionine and methionine absorption in the intestinal tract of green sturgeon (Acipenser medirostris). Aquat Toxicol 96, 62-69.

37. Vendeland SC, Deagen JT, Butler JA, et al. (1994) Uptake of selenite, selenomethionine and selenate by brush border membrane vesicles isolated from rat small intestine. Biometals 7, 305-312.

38. Nickel A, Kottra G, Schmidt G, et al. (2008) Characteristics of transport of selenoamino acids by epithelial amino acid transporters. Chem Biol Interact 177, 234-241. 